\title{
Telemedicine curriculum in undergraduate medical education: a systematic search and review
}

\author{
Işıl İrem Budakoğlu' ${ }^{1}$ Mustafa Ünal Sayııır ${ }^{2}$ - Yavuz Selim Kıyak ${ }^{1}$ - Özlem Coşkun ${ }^{1}$ Serdar Kula ${ }^{3,4}$
}

Received: 6 April 2021 / Accepted: 5 May 2021 / Published online: 10 May 2021

(c) IUPESM and Springer-Verlag GmbH Germany, part of Springer Nature 2021

\begin{abstract}
The number of patient-doctor appointments carried out using telemedicine has surpassed in-person appointments. In spite of this, it is unclear that telemedicine curricula in undergraduate medical education reflect the real importance by means of the effectiveness of these approaches. We aimed to systematically search and review the studies that are on undergraduate telemedicine curricula. We searched the Web of Science, PubMed, and Scopus using the keywords such as telemedicine, medical education, and curriculum. Our search was limited to publication dates between January 1, 2000, and February 1, 2020. We elicited the information of the curricula as to their countries, participants, aims or objectives, teaching methods, and evaluation of effectiveness. We also evaluated the quality of the studies using the Joanna Briggs Institute Qualitative Appraisal and Review Instrument. Out of 461 studies, seven articles were selected based on selection criteria for further review. The studies were mostly from the USA. The participant numbers were between seven and 268 . There were several modes of delivery but lectures and patient encounters were used mostly. In four studies, the effectiveness was evaluated only by using satisfaction surveys, and the results were satisfactory. A study reported the acquisition and application of skills as a result. There is no well-established telemedicine curriculum in the undergraduate years. The methods vary but the effectiveness of the educational programs does not have a robust evidence base. It is evident that undergraduate medical education needs a curriculum backed by strong scientific data on its effectiveness.
\end{abstract}

Keywords Telemedicine $\cdot$ Curriculum $\cdot$ Medical education $\cdot$ Undergraduate

\section{Introduction}

Telemedicine is a subset of telehealth and it refers to the use of communication networks to deliver healthcare services at a distant [1]. World Health Organization (WHO) defines telemedicine as "the delivery of health care services, where distance is a critical factor, by all health care professionals using information and communication technologies for the exchange of valid information for diagnosis, treatment and

Iş11 İrem Budakoğlu

isilirem@gazi.edu.tr

1 Department of Medical Education and Informatics, Faculty of Medicine, Gazi University, Ankara, Turkey

2 Faculty of Medicine, Gazi University, Ankara, Turkey

3 Department of Informatics, Institute of Informatics, Gazi University, Ankara, Turkey

4 Department of Pediatric Cardiology, Faculty of Medicine, Gazi University, Ankara, Turkey prevention of disease and injuries, research and evaluation, and for the continuing education of health care providers, all in the interests of advancing the health of individuals and their communities" [2]. The history of telemedicine started with medical assistance call about spilling acid on Alexander Graham Bell's trousers [3] ends up carrying out more patient-doctor appointments by means of telemedicine than in person [4].

Today, healthcare centers are making more use of the power and possibilities of information technologies to support patient-centered and individualized forms of healthcare. This increases the interest in using telemedicine in health institutions. Using telemedicine in medical practices allows healthcare providers to contact patients remotely [5] and enables healthcare authorities to manage challenges such as a shortage of healthcare workers especially in rural areas [1]. The technological tools that are used for telemedicine have a broad range from simple e-mails to complex robotic surgery [1]. The communication and networking technologies also vary such as satellite communications, internet, 
Global System for Mobile Communications (GSM) [1]. The COVID-19 pandemics led to such a need for sociallydistanced health care that telemedicine visits increased 683\% at the height of the pandemic [6]. It seems that telemedicine will permeate pervasively into healthcare delivery over time [7].

According to an economic forecast organization, the size of the telemedicine market was around $\$ 45$ billion in 2019 and it is projected to reach $\$ 175$ billion by 2026 [8]. The reasons for this growth are the increasing healthcare cost, geriatric population, government funding and grants for telemedicine, medical tourism, prevalence of chronic and lifestyle-associated diseases, medical requirement in remote areas, and need for remote patient monitoring services in developing countries [8]. In the European Union, five projects related to telemedicine are carried out [9]. The scope of these projects is teleradiology, teledermatology, teleneurology, telemonitoring for diabetes and chronic heart failure, but there is no topic on education [10]. It was reported in the same report that medical schools did not fully include telemedicine education in their curriculum. As today's medical students have grown up using digital technology, they will not have much trouble integrating these skills into medical care delivery. Although students have good digital literacy, formal and structured education is still needed to ensure they provide high-quality telemedical care [11]. Given the essential need for early clinical experiences to help medical students cultivate their skills [12], it follows that early implementation of telemedicine training at the undergraduate level will prepare future physicians for the telemedicine universe. In support of this argument, data suggest that medical students feel more benefit from teledermatology training than residents [13]. Germany has begun to address this issue and has developed a five-module education at the University of Mainz Medical School aimed at improving students' skills in the field of telemedicine. In France, telemedicine has been integrated into the medical curriculum since 2009 [14]. The American Medical Association (AMA) reported that medical students and residents should learn to use telemedicine in clinical practice. For this purpose, accreditation bodies particularly encourage them to include core competencies for telemedicine in both undergraduate and postgraduate medical education programs [15].

In spite of its importance, there is a limited number of systematic reviews about telemedicine education, either in general or at the undergraduate level in particular. One of them is a mixed-methods review focused on the undergraduate level, limited to the United States [16]. The other study determines the extent of medical students' and residents' exposure to telemedicine and is limited to medical schools in the United States [17]. Three other studies concerning telemedicine education and training do not focus on the undergraduate level. Two of these address training for all health professions $[18,19]$, while another includes all periods of medical education [20].

Nevertheless, there is a significant gap in the literature for systematic reviews of telemedicine in undergraduate medical education. To fill this gap, we aimed to carry out a systematic search and literature review that focuses on just undergraduate medical education curriculum of telemedicine. The research questions are as follows:

- Which educational years/level have been the telemedicine curriculum implemented?

- What are the aims or objectives of the telemedicine curriculum?

- What are the methods used to teach telemedicine?

- How effective is the telemedicine curriculum?

\section{Materials and methods}

\subsection{Study design}

We conducted a systematic database review of published studies in the past 20 years (2000-2020), in order to identify literature regarding telemedicine curriculum in undergraduate medical education, we followed the preferred reporting items for systematic reviews and meta-analyses (PRISMA) guidelines [21]. PRISMA is the minimum set of evidence-based items for reporting in systematic reviews and meta-analyzes. It focuses on randomized trials, but can also be used as a basis for reporting evaluations of other types of research.

\subsection{Search strategy and inclusion criteria}

We searched the terms "telemedicine", "medical education", "curriculum" in the Web of Science, PubMed, and Scopus databases. We used Boolean connectors/operators, to get more precise and more relevant results in searches. The inclusion criteria that should be met were the following: Participants are medical undergraduates, and intervention is telemedicine curriculum/course.

Two reviewers screened the retrieved articles based on the inclusion criteria. Duplicates and studies unrelated to telemedicine curriculum were excluded. After this process, the other two reviewers independently screened the abstracts of the remaining studies. Studies that seemed unclear from an abstract screening alone were evaluated by a reading of the full-text. Conflicts between reviewers were resolved through consensus. 
Table 1 Screening and exclusion the studies according to databases

\begin{tabular}{lllll}
\hline Database & \multicolumn{3}{l}{ Number of documents } & When duplicates are excluded \\
\cline { 2 - 5 } & $\begin{array}{l}\text { Studies } \\
\text { screened }\end{array}$ & $\begin{array}{l}\text { Full-text assess- } \\
\text { ment }\end{array}$ & Included & 7 \\
\hline Web of Science & 50 & 24 & 4 & \\
PubMed & 167 & 45 & 12 & \\
Scopus & 244 & 35 & 11 & \\
Total & 461 & 104 & 27 & \\
\hline
\end{tabular}

\subsection{Quality assessment}

In reviewing the quality of telemedicine articles, we followed the methodology of Edirippulige and Armfield.18 Like them, we applied the Critical Appraisal checklist for Interpretive and Critical Research, based on the Joanna Briggs Institute Qualitative Appraisal and Review Instrument (JBI QARI) [22].

Fig. 1 PRISMA diagram

\section{Results}

\subsection{General results of systematic review}

Table 1 and Fig. 1 show the exclusion process and the number of the studies screened, included, and excluded. Our initial search for publications involving keywords resulted in a total of 461 articles. After the abstract review, the full

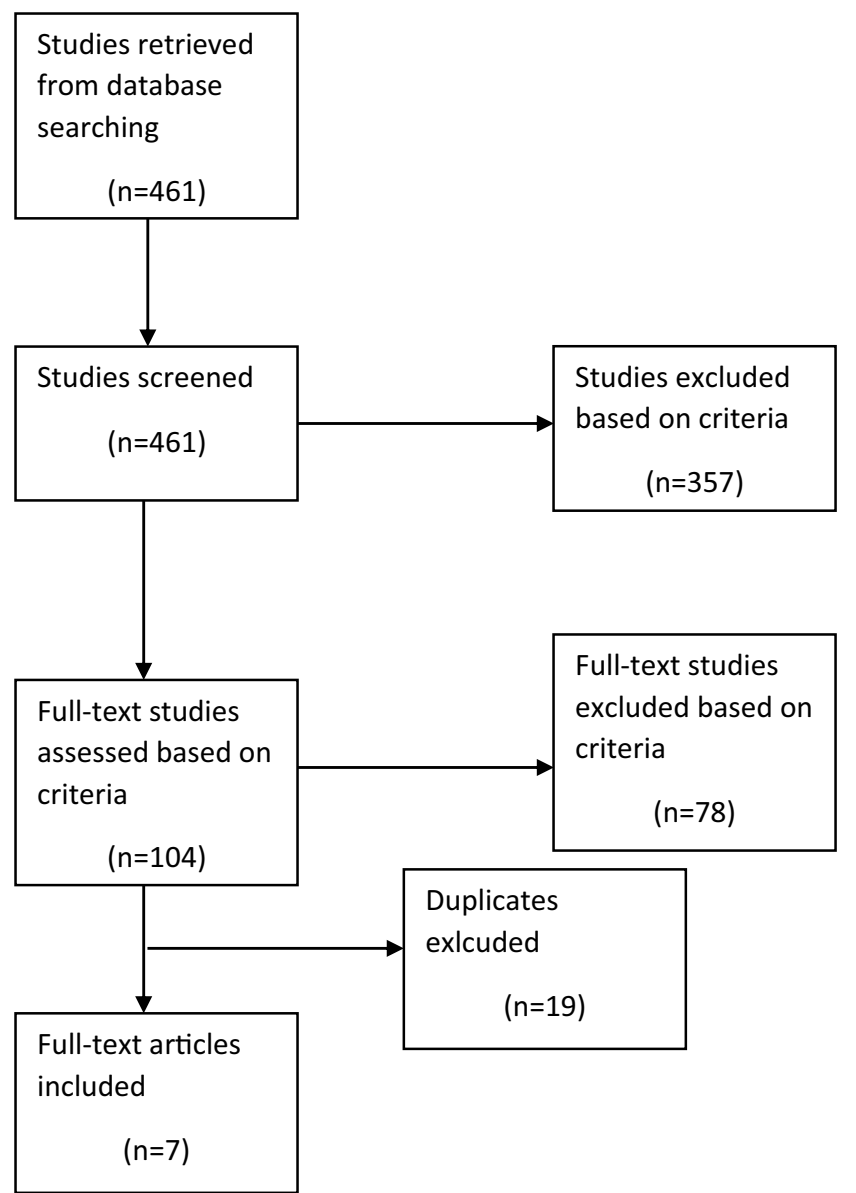


text of 34 articles was reached. Although the articles contain keywords, a total of 28 articles were identified for undergraduate telemedicine curriculum. When the same articles in all three databases were removed, the remaining 7 articles were examined.

Out of seven studies, five were published in 2016 or after; one in 2010 [23]; and another in 2014 [24]. Four studies were from the USA, two from Australia, and one from Switzerland.

Assessment of the quality of the studies showed that quality scores totaled seven for two studies [25, 26] (Table 2). In one of the studies, Jonas et al. [25] offered a learner-centered telemedicine curriculum for 149 third-year medical students. Firstly, they reported an asynchronous lecture to introduce covering topics such as telehealth history, application, and ethics. Following the lectures, they carried out interactive telehealth instruction sessions, mock patient telehealth encounters, and hands-on practice using telehealth equipment. They conducted a pretest and a posttest comprising multiple-choice questions. They demonstrated that $\% 10.1$ improvement in test scores. The other study that had the best quality score was a randomized crossover trial [26]. In this study, Liu et al. [26] reported the trial results of a telehealth communication skills training platform, which is called as EQClinic, incorporated into the undergraduate curriculum. Even if the focus of the study is to compare the effectiveness of face-to-face and teleconsultation in terms of teaching communication skills, the study includes a telehealth intervention in a curriculum. The results of the study showed that conducting teleconsultations through EQClinic improved medical communication skills of the students.

Table 3 presents the study details for the answers to the research questions.

\subsubsection{The year/level the telemedicine curriculum was implemented}

The curriculum in the studies was implemented for the second, third, or fourth year medical students or some combinations of them.

\subsubsection{The aims or objectives of telemedicine curriculum}

Out of seven studies, four articles focused mainly on teaching how telemedicine practice works. The other studies integrated telemedicine into the curriculum (1) to teach communication skills [26], (2) to compare telepresence training and in-person training [27], and (3) to teach ethics [24].

\subsubsection{The methods used to teach telemedicine}

Four out of seven studies used lecture as a delivery method $[23,24,28,29]$. The lectures mostly were to introduce the

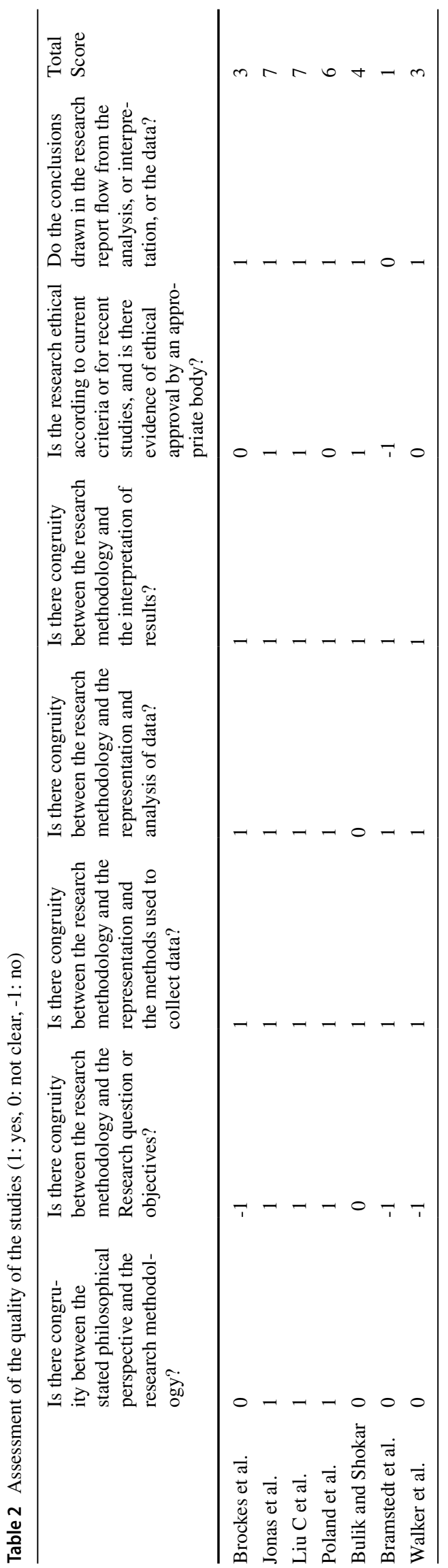




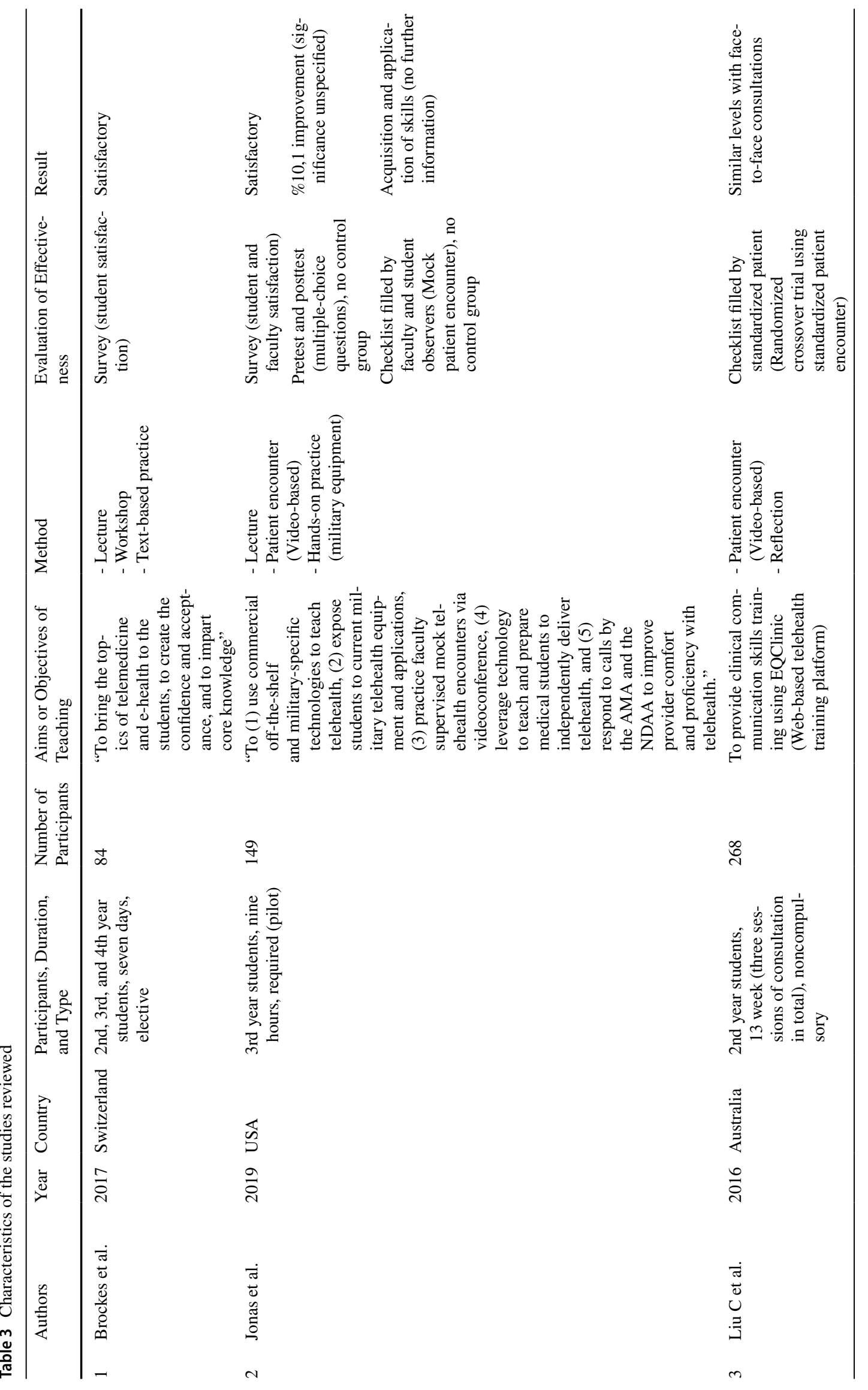




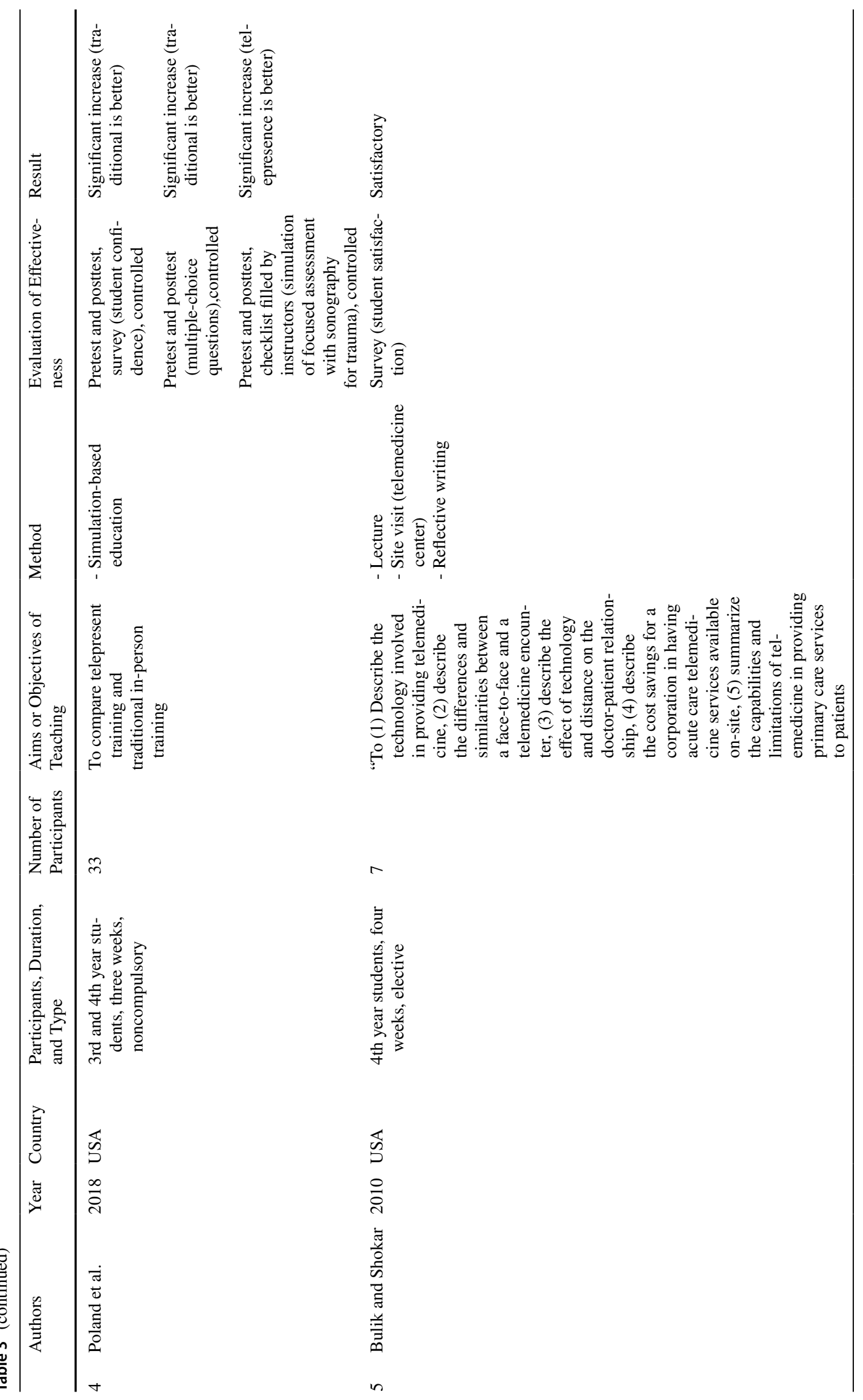




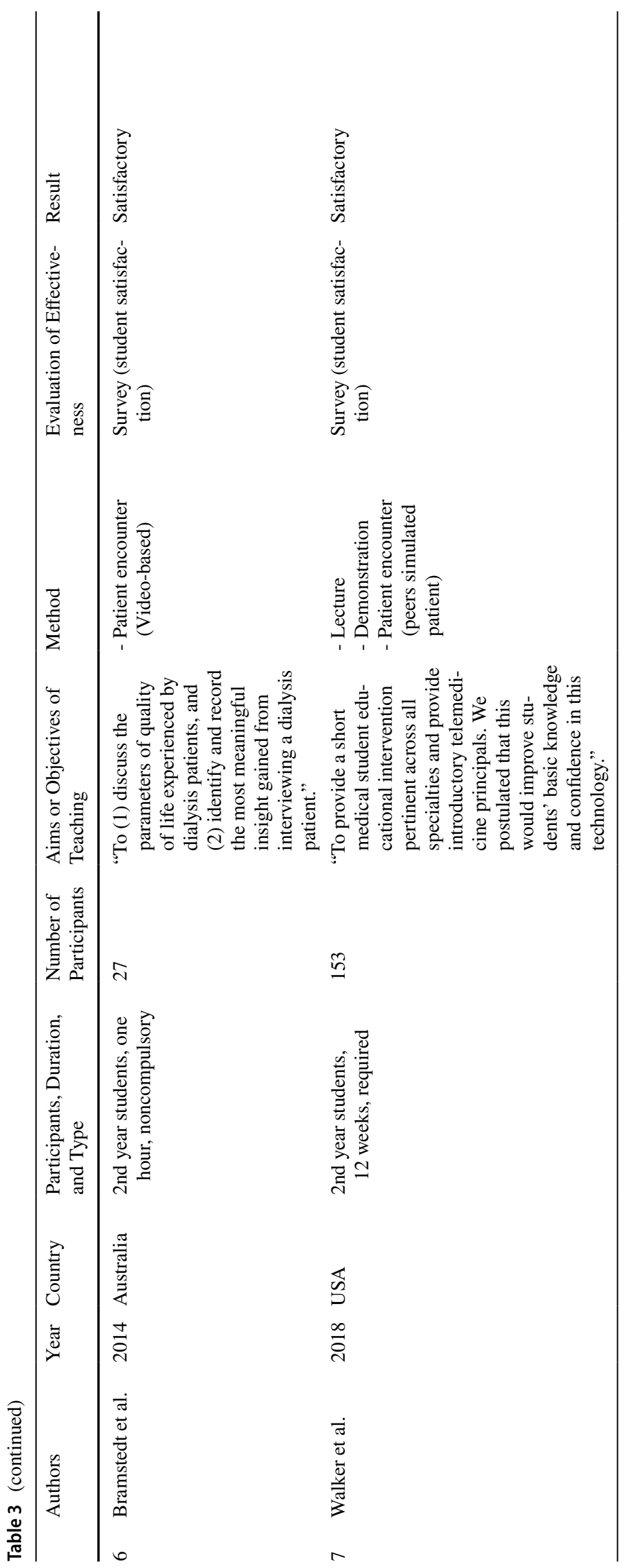


basic concepts related to telemedicine. Patient encounter was another prominent method and it was used in four studies. Out of these four studies, three of them carried out the patient encounters using video-based communication [24-26]. In another, the patient encounter was conducted as an in-class simulation and the simulated patients were the peers of the students [29]. The other methods were textbased practice [28], workshop [28], visiting a center [23], reflective writing [23], reflection [26], simulation-based education [27], and demonstration [29]. The duration of the courses varied from one hour [24] to 13 weeks [26]. Participant numbers in the studies were between seven [23] and $268[26]$.

\subsubsection{Effectiveness of the telemedicine curriculum}

To determine effectiveness, we conducted surveys for six studies [23-25, 27-29]. A satisfaction survey served as the only source of information on effectiveness for four studies $[23,24,28,29]$. While there was a control group in one study [27], another study carried out a randomized crossover trial [26]. Surveys showed that the courses were satisfactory from the perspective of students [23-25, 27-29], and faculty members [25]. It was shown that there was $10.1 \%$ improvement [25] and significant increase [27] between pretest and posttest in multiple-choice questions. Two studies showed an increase in terms of improvement in patient encounter skills; one reported "acquisition and application of skills" [25], and another showed developing skills of similar levels with face-to-face consultations [26].

\section{Discussion}

In this study, we aimed to review the undergraduate curriculum of telemedicine. We found a limited number of teaching interventions in the curriculum in a limited number of countries. Today, the pandemic problem experienced by the whole world has revealed the importance of telemedicine in health service delivery. It is now a necessity for doctors to graduate ready to provide services via telemedicine. However, studies were limited to only three countries from all over the world. The studies mostly were from the USA [23, $25,27,29]$. The reason behind this would be the fact that "USA prioritizes to incorporate telemedicine into healthcare system" [30]. Interestingly, the study from Switzerland introduced a curriculum that dates back to 2008 that shows years of experience [28].

Today's modern medical education strategy supports the view that the topics should be spread over all years of education in an integrated manner [31]. Undergraduate years of medical education are essential to develop the skills that students need to use in their professional lives [32]. Our findings show that telemedicine curricula in undergraduate education occur mostly in the second through fourth years. Considering the high degree of student satisfaction in the studies [23-25, 27-29], it seems that telemedicine education in undergraduate years is acceptable to medical students. In particular, they state that hands-on practice is very helpful to develop their telemedicine skills.

The current review found that there are plenty of methods that were used to teach telemedicine. Two prominent methods are lectures and patient encounters, which are similar to telemedicine teaching in postgraduate and continuous medical education [20]. While lectures are useful to introduce basic concepts, patient encounters provide fruitful opportunities to apply the knowledge. Given that adults learn better by engaging in hands-on practice [33], using video-based patient encounters to teach telemedicine would be seen as a wise choice. Regardless of which method is used, however, it should be shown that there is scientific evidence for the effectiveness of that method.

More than half of the studies we analyzed provided evidence on effectiveness just by measuring student satisfaction $[23,24,28,29]$. However, it is well-known that examining learner satisfaction alone provides very limited evidence about the effectiveness of a curriculum [34]. Knowledge gained, behavior changed, and long-term effects are more robust to prove the effectiveness of a curriculum [34]. Two studies $[25,26]$, for example, suggest that interventions highlighting patient encounters as an assessment strategy changed behavior.

The main findings of this review would be that there is a limited number of training opportunities to learn telemedicine in undergraduate years, and these approaches are not backed by solid data on effectiveness. These findings are similar to the results of the systematic review of telehealth-related academic or vocational education [18]. Lack of telemedicine training is a concern for the USA as well [17], and lack of evidence-based approaches is a concern all across the world even in postgraduate years [20].

Our study has limitations. We included studies published only up to February 1, 2020, after which the huge increase in demand for telemedicine during the COVID-19 pandemic [7] could have led to an increase in studies about teaching telemedicine in undergraduate years. These publications did not take place in this review. Because of the timing of our research, we did not review studies published after the global proliferation of COVID-19 cases. Another limitation is the possibility that there are more published curricula on telemedicine between the dates we reviewed. To not overlook any study, we used broad keywords and then evaluated all of the abstracts.

\section{Conclusions}

The overall picture of this review shows us there is not any well-established telemedicine curriculum in undergraduate medical education. There are several approaches but most 
of them provide weak evidence about their effectiveness. Due to the fact that telemedicine will continue to permeate excessively into healthcare delivery [7], it is evident that medical schools around the world need more approaches to establish telemedicine curriculum in undergraduate years that are backed by robust scientific data on the effectiveness.

Acknowledgements The authors acknowledge Kadriye O Lewis, Ed.D. Professor, for her feedback and input.

Funding The authors received no financial support for the study.

\section{Declarations}

Ethical approval As there were no human participants involved, ethical approval and consent were not required to obtain for this study.

Conflict of interest The authors declare that there is no conflict of interest.

\section{References}

1. Sood S, Mbarika V, Jugoo S, et al. What is telemedicine? A collection of 104 peer-reviewed perspectives and theoretical underpinnings. Telemed J E Health. 2007;13:573-590.

2. World Health Organization. Telemedicine: Opportunities and developments in member states. 2010. https://www.who.int/goe/publications/ goe_telemedicine_2010.pdf Accessed 29 June 2020.

3. Aronson SH. The Lancet on the telephone 1876-1975. Med Hist. 1977;21:69-87.

4. Owens B. Telemedicine on the rise but lagging in Canada. CMAJ. 2018;190(38):E1149-50.

5. Gogia S. Rationale, history, and basics of telehealth. In: Gogia S, editor. Fundamentals of Telemedicine and Telehealth. 1st ed. London: Academic Press; 2019. p. 11-34.

6. Mann DM, Chen J, Chunara R, et al. COVID-19 transforms health care through telemedicine: Evidence from the field. J Am Med Inform Assoc. 2020;27:1132-5.

7. Wosik J, Fudim M, Cameron B, et al. Telehealth Transformation: COVID-19 and the rise of Virtual Care. J Am Med Inform Assoc. 2020;27:957-62.

8. CNBC. How coronavirus accelerated the rise of telemedicine. 2020. www.cnbc.com/2020/05/18/coronavirus-how-covid-19-acceleratedthe-rise-of-telemedicine.html Accessed 29 June 2020.

9. European Comission. Market study on telemedicine. 2018. https:// ec.europa.eu/health/sites/health/files/ehealth/docs/2018_provision_ marketstudy_telemedicine_en.pdf Accessed 10 August 2020.

10. European Comission. Market study on telemedicine. 2018. p.22. https://ec.europa.eu/health/sites/health/files/ehealth/docs/2018_ provision_marketstudy_telemedicine_en.pdf 10 August 2020.

11. European Comission. Market study on telemedicine. 2018. pp. 97-98. https://ec.europa.eu/health/sites/health/files/ehealth/docs/ 2018_provision_marketstudy_telemedicine_en.pdf 10 August 2020.

12. Littlewood S, Ypinazar V, Margolis SA, et al. Early practical experience and the social responsiveness of clinical education: systematic review. BMJ. 2005;331:387-91.

13. Boyers LN, Schultz A, Baceviciene R, et al. Teledermatology as an educational tool for teaching dermatology to residents and medical students. Telemed J E Health. 2015;21:312-4.

14. Yaghobian S, Ohannessian R, Mathieu-Fritz A, et al. National survey of telemedicine education and training in medical schools in France. J Telemed Telecare. 2020;26:303-8.
15. American Medical Association. AMA encourages telemedicine training for medical students, residents. 2016. https://www.ama-assn.org/ press-center/press-releases/ama-encourages-telemedicine-trainingmedical-students-residents Accessed 27 March 2021.

16. Waseh S, Dicker AP. Telemedicine training in undergraduate medical education: mixed-methods review. JMIR Med Educ. 2019;5(1):e12515.

17. Pourmand A, Ghassemi M, Sumon K, et al. Lack of telemedicine training in academic medicine: are we preparing the next generation? Telemed J E Health. 2021;27(1):62-7.

18. Edirippulige S, Armfield NR. Education and training to support the use of clinical telehealth: a review of the literature. J Telemed Telecare. 2017;23(2):273-82.

19. Chike-Harris KE, Durham C, Logan A, et al. Integration of Telehealth Education into the Health Care Provider Curriculum: A Review. Telemed J E Health. 2021;27(2):137-49.

20. Stovel RG, Gabarin N, Cavalcanti RB, et al. Curricular needs for training telemedicine physicians: a scoping review. Med Teach. 2020;42:1234-42.

21. Liberati A, Altman DG, Tetzlaff J, et al. The PRISMA statement for reporting systematic reviews and meta-analyses of studies that evaluate health care interventions: explanation and elaboration. $\mathrm{J}$ Clin Epidemiol. 2009;62:e1-34.

22. Joanna Briggs Institute. JBI QARI critical appraisal checklist for interpretive \& critical research. 2019. https://jbi.global/sites/ default/files/2019-05/JBI_Critical_Appraisal-Checklist_for_ Qualitative_Research2017_0.pdf Accessed 29 June 2020.

23. Bulik RJ, Shokar GS. Integrating telemedicine instruction into the curriculum: expanding student perspectives of the scope of clinical practice. J Telemed Telecare. 2010;16(7):355-8.

24. Bramstedt KA, Prang M, Dave S, et al. Telemedicine as an ethics teaching tool for medical students within the nephrology curriculum. Prog Transplant. 2014;24:294-7.

25. Jonas CE, Durning SJ, Zebrowski C, et al. An interdisciplinary, multi-institution telehealth course for third-year medical students. Acad Med. 2019;94:833-7.

26. Liu C, Lim RL, McCabe KL, et al. A web-based telehealth training platform incorporating automated nonverbal behavior feedback for teaching communication skills to medical students: a randomized crossover study. J Med Internet Res. 2016;18:e246.

27. Poland S, Frey JA, Khobrani A, et al. Telepresent focused assessment with sonography for trauma examination training versus traditional training for medical students: a simulation-based pilot study. J Ultrasound Med. 2018;37:1985-92.

28. Brockes C, Grischott T, Dutkiewicz M, et al. Evaluation of the education "Clinical Telemedicine/e-Health" in the curriculum of medical students at the University of Zurich. Telemed J E Health. 2017;23:899-904.

29. Walker C, Echternacht H, Brophy PD. Model for medical student introductory telemedicine education. Telemed J E Health. 2019;25(8):717-23.

30. Rao B, Lombardi A. Telemedicine: current status in developed and developing countries. J Drugs Dermatol. 2009;8(4):371-5.

31. Harden RM. The integration ladder: a tool for curriculum planning and evaluation. Med Educ. 2000;34:551-7.

32. Dornan T, Littlewood S, Margolis SA, et al. How can experience in clinical and community settings contribute to early medical education? A BEME systematic review Med Teach. 2006;28(1):3-18.

33. Taylor DC, Hamdy H. Adult learning theories: Implications for learning and teaching in medical education: AMEE Guide No. 83. Med Teach. 2013;35(11):e1561-e1572.

34. Frye AW and Hemmer PA. Program evaluation models and related theories: AMEE guide no. 67. Med Teach. 2012;34(5):e288-e299. 